\title{
The permanence and periodic solution of a competitive system with infinite delay, feedback control, and Allee effect
}

\author{
Lei Shi ${ }^{1}$, Hua Liu ${ }^{1 *}$ (D), Yumei Wei ${ }^{2}$ Ming Ma and Jianhua Ye
}

\section{"Correspondence:}

7783360@qq.com

${ }^{1}$ School of Mathematics and Computer Science, Northwest Minzu University, Lanzhou, People's Republic of China

Full list of author information is available at the end of the article

\begin{abstract}
This paper is devoted to study the permanence and periodic solution of a competitive system with infinite delay, feedback control, and the Allee effect. We derive sufficient conditions for the permanence and existence of a periodic solution in a competitive system with infinite delay, feedback control, and the Allee effect by using the differential inequality theory and constructing the Lyapunov function. We provide explicit estimates of the lower and upper bounds of the population density. This study reveals that the Allee effect plays an essential role in the permanence and increases the risk of population extinction.
\end{abstract}

MSC: 34A40; 34B15; $92 \mathrm{~B} 05$

Keywords: Permanence; Allee effect; Infinite delay; Feedback control; Periodic solution

\section{Introduction}

A basic question of theoretical and practical importance in population biology concerns the long-term survival of each species. Permanence, addressing the long-term survival of each component of a system, has emerged as the most important notion for the systems in ecology. Ahmad [1] studied the permanence of the following traditional two-species nonautonomous Lotka-Volterra system

$$
\left\{\begin{array}{l}
x_{1}^{\prime}(t)=x_{1}(t)\left[a_{1}(t)-b_{11}(t) x_{1}(t)-b_{12}(t) x_{2}(t)\right] \\
x_{2}^{\prime}(t)=x_{2}(t)\left[a_{2}(t)-b_{21}(t) x_{1}(t)-b_{22}(t) x_{2}(t)\right]
\end{array}\right.
$$

The functions $a_{k}(t)$ and $b_{k j}(t)(1 \leq k, j \leq 2)$ defined on $(-\infty,+\infty)$ are positive continuous upper bounded and have positive lower bounds. Letting

$$
g_{L}\left(g_{M}\right)=\inf (\sup )\{g(t): t \in R\}
$$

for each bounded function $g: R \rightarrow R$, he showed that one of them will be driven to extinction whereas the other will stabilize at a certain solution to the corresponding logistic

(c) The Author(s) 2018. This article is distributed under the terms of the Creative Commons Attribution 4.0 International License (http://creativecommons.org/licenses/by/4.0/), which permits unrestricted use, distribution, and reproduction in any medium, provided you give appropriate credit to the original author(s) and the source, provide a link to the Creative Commons license, and indicate if changes were made. 
equations if the inequalities

$$
a_{1 L}>b_{12 M} \frac{a_{2 M}}{b_{22 L}}, \quad a_{2 M} \leq b_{21 L} \frac{a_{1 L}}{b_{11 M}}
$$

hold, that is, there can be no coexistence of the two species.

Under the above conclusion, the permanence of the traditional two-species LotkaVolterra system with finite delays is also extended and attracts more and more attention to mathematics and mathematical biology (Teng [2-4], Zhao and Jiang [5], Chen [6, 7], Lisena [8], and Muroya [9]).

Oca and Vivas [10] investigated the dynamic behavior of the two-species Lotka-Volterra system of integro-differential equations with infinite delay

$$
\left\{\begin{array}{l}
x_{1}^{\prime}(t)=x_{1}(t)\left[a_{1}(t)-b_{11}(t) x_{1}(t)-b_{12}(t) \int_{-\infty}^{t} k_{1}(t-s) x_{2}(s) d s\right] \\
x_{2}^{\prime}(t)=x_{2}(t)\left[a_{2}(t)-b_{21}(t) \int_{-\infty}^{t} k_{2}(t-s) x_{1}(s) d s-b_{22}(t) x_{2}(t)\right]
\end{array}\right.
$$

The parameters are the same as in model (1.1). Oca and Vivas showed that if (1.2) holds, then the results of [1] still hold for system (1.3) by applying the fluctuation theorem (Tieno [11] or Hirsch et al. [12]).

The feedback control method of population ecosystem is applied, and many scholars have studied the stability and persistent adaptation of the ecosystem with feedback control to accelerate the degraded ecosystem to the equilibrium state or to adjust the equilibrium state to a new position. Xiao et al. [13] had proposed the following two-species competitive system with feedback controls:

$$
\left\{\begin{array}{l}
x_{1}^{\prime}(t)=x_{1}(t)\left[a_{1}(t)-b_{11}(t) x_{1}(t)-b_{12}(t) x_{2}(t)-c_{1}(t) u_{1}(t)\right] \\
x_{2}^{\prime}(t)=x_{2}(t)\left[a_{2}(t)-b_{21}(t) x_{1}(t)-b_{22}(t) x_{2}(t)+c_{2}(t) u_{2}(t)\right], \\
u_{1}^{\prime}(t)=-e_{1}(t) u_{1}(t)+d_{1}(t) x_{1}(t) \\
u_{2}^{\prime}(t)=f(t)-e_{2}(t) u_{2}(t)-d_{2}(t) x_{2}(t) .
\end{array}\right.
$$

The functions $a_{i}(t), b_{i j}(t), e_{i}(t), d_{i}(t), f_{i}(t)(i, j=1,2)$ are positive continuous upper bounded and have positive lower bounds.

According [14], for any positive solution $\left(x_{1}(t), x_{2}(t)\right)$ of system (1.3), we have $x_{2}(t) \rightarrow 0$ as $t \rightarrow+\infty$ and $x_{1}(t)-x^{*}(t) \rightarrow 0$ as $t \rightarrow+\infty$, where $x^{*}(t)$ is the solution of some logistic equation. However, this result breaks the equilibrium state of the two-species competitive system, so it needs to be adjusted by feedback control to make the ecosystem rebalance.

It is well known that the role of feedback control is enabling the ecosystem to reach and maintain equilibrium or steady state, and the result of feedback is to suppress and attenuate the changes that occur to the originally changed component.

In [13], the authors searched for certain schemes (such as harvesting procedure) to ensure the system to coexist with condition (1.2), where $c_{2}(t) u_{2}(t)$ indicates the size to be used to make the population $x_{2}(t)$ not extinct, and $c_{2}(t)$ is the proportional coefficient of input; meanwhile, $c_{1}(t) u_{1}(t)$ is the size that reduces the number of $x_{1}(t)$ by periodic capture, and $c_{1}(t)$ is the proportional coefficient capture. Population $u_{1}(t)$ has a negative growth rate of a proportional coefficient $e_{1}(t)$; we adjust it by the proportional coefficient $d_{1}(t)$ to avoid extinction; $f(t)$ is the initial population size of $u_{2}(t), e_{2}(t)$ acts in the same way as $e_{1}(t)$, and $d_{2}(t)$ has the same effect as $d_{1}(t)$. 
They showed that assuming, in addition to (1.2), that

$$
\begin{aligned}
& a_{1 L}-\frac{a_{2 M} b_{12 M}}{b_{22 L}}>\frac{c_{1 M} a_{1 M} d_{1 M}}{e_{1 L} b_{11 L}}+\frac{b_{12 M} c_{2 M} f_{M}}{b_{22 L} e_{2 L}}, \\
& \frac{c_{2 L}}{e_{2 M}}\left[f_{L}-\frac{d_{2 M} a_{2 M} e_{2 L}+d_{2 M} c_{2 M} f_{M}}{b_{22 L} e_{2 L}}\right]>\frac{a_{1 M} b_{21 M}}{b_{11 L}}-a_{2 L},
\end{aligned}
$$

system (1.4) is permanent. In other words, by selecting appropriate feedback control variables, the extinction of species can be kept. We easily find that the additional work associated with the ecosystem with feedback controls is improved and extended for the present paper; see [15-27].

In addition, the ecological system is usually affected by seasonal factors, so that the system parameters show the changing characteristics of the cycle. Therefore, it is of great theoretical and practical significance to consider the ecosystem with feedback control and infinite delay effect and to study its stability and durability. Chen [14] considers the following two-species competitive system with feedback controls and infinite delay:

$$
\left\{\begin{aligned}
x_{1}^{\prime}(t)= & x_{1}(t)\left[a_{1}(t)-b_{11}(t) x_{1}(t)-b_{12}(t) \int_{-\infty}^{t} k_{1}(t-s) x_{2}(s) d s\right. \\
& \left.-c_{1}(t) \int_{-\infty}^{t} h_{1}(t-s) u_{1}(s) d s\right] \\
x_{2}^{\prime}(t)= & x_{2}(t)\left[a_{2}(t)-b_{21}(t) \int_{-\infty}^{t} k_{2}(t-s) x_{1}(s) d s-b_{22}(t) x_{2}(t)\right. \\
& \left.+c_{2}(t) \int_{-\infty}^{t} h_{2}(t-s) u_{2}(s) d s\right] \\
u_{1}^{\prime}(t)= & -e_{1}(t) u_{1}(t)+d_{1}(t) \int_{-\infty}^{t} k_{3}(t-s) x_{1}(s) d s \\
u_{2}^{\prime}(t)= & f(t)-e_{2}(t) u_{2}(t)-d_{2}(t) \int_{-\infty}^{t} k_{4}(t-s) x_{2}(s) d s .
\end{aligned}\right.
$$

The parameters in this model are the same as in model (1.4). The delay kernels are nonnegative measurable, where

$$
\int_{0}^{+\infty} k_{i}(s) d s=1, \quad \int_{0}^{+\infty} h_{i}(s) d s=1,
$$

and

$$
k_{i}:[0,+\infty) \rightarrow(0,+\infty), \quad i=1, \ldots, 4 ; \quad h_{i}:[0,+\infty) \rightarrow(0,+\infty), \quad i=1,2 .
$$

They showed that system (1.6) is permanent if there are positive constants $\alpha, \beta$ such that

$$
\begin{aligned}
& 0<\alpha \leq \lim \inf _{t \rightarrow+\infty} x_{i}(t) \leq \lim \sup _{t \rightarrow+\infty} x_{i}(t) \leq \beta<+\infty, \\
& 0<\alpha \leq \lim \inf _{t \rightarrow+\infty} u_{i}(t) \leq \lim \sup _{t \rightarrow+\infty} u_{i}(t) \leq \beta<+\infty,
\end{aligned}
$$

and (1.2) and (1.5) are satisfied.

On the other hand, Allee effects are phenomena in population biology that arise if percapita growth rates increase initially with increasing population sizes or densities. The phenomena were first observed by Allee in the 1930s in [28-30] and have received considerable attention recently due to fragmentation of habitats, invasion of exotic species, 
biology control of pest, and so on. A corresponding result of a different Allee effect form is presented in [31-33]. Moreover, some scholars have been committed to studying the effect of Allee effect on the persistence of competition systems with infinite delay and feedback control.

In this paper, the most representative expression of Allee effect is $\left(1-\frac{A+c}{x_{1}(t)+c}\right)$ [31, 32], where $A$ is the Allee threshold, $A>0$ means the strong Allee effect, and $A<0$ means the feeble Allee effect, $c$ is an auxiliary parameter $(c>0, c \geq-A)$; when $A+c=0$, the Allee effect expression value 1 indicates that the species has no Allee effect. The parameter $c$ affects the growth curve of species, which flattens out as it increases. When $x_{1}(t)<A$, that is, the population density of species is less than $A$, the Allee effect is less than zero, and the population grows negatively; conversely, when $x_{1}(t)>A$, the population density of species is greater than $A$, the Allee effect is greater than zero, and the population shows a positive growth trend.

This analysis motivated us to propose the following two-species competitive system with infinite delay, feedback, and Allee effect:

$$
\left\{\begin{aligned}
x_{1}^{\prime}(t)= & x_{1}(t)\left[a_{1}(t)\left(1-\frac{b_{11}(t)}{a_{1}(t)} x_{1}(t)\right)\left(1-\frac{A+c}{x_{1}(t)+c}\right)-b_{12}(t) \int_{-\infty}^{t} k_{1}(t-s) x_{2}(s) d s\right. \\
& \left.-c_{1}(t) \int_{-\infty}^{t} h_{1}(t-s) u_{1}(s) d s\right], \\
x_{2}^{\prime}(t)= & x_{2}(t)\left[a_{2}(t)-b_{21}(t) \int_{-\infty}^{t} k_{2}(t-s) x_{1}(s) d s-b_{22}(t) x_{2}(t)\right. \\
& \left.+c_{2}(t) \int_{-\infty}^{t} h_{2}(t-s) u_{2}(s) d s\right] \\
u_{1}^{\prime}(t)= & -e_{1}(t) u_{1}(t)+d_{1}(t) \int_{-\infty}^{t} k_{3}(t-s) x_{1}(s) d s \\
u_{2}^{\prime}(t)= & f(t)-e_{2}(t) u_{2}(t)-d_{2}(t) \int_{-\infty}^{t} k_{4}(t-s) x_{2}(s) d s .
\end{aligned}\right.
$$

The parameters are the same as in model (1.6). We consider (1.9) together with the initial conditions

$$
\begin{aligned}
& x_{i}(s)=\phi_{i}(s), \quad s \in(-\infty, 0], i=1,2, \\
& u_{i}(s)=\psi_{i}(s), \quad s \in(-\infty, 0], i=1,2,
\end{aligned}
$$

where $\phi_{i}, \psi_{i} \in B C^{+}$, and

$$
B C^{+}=\{\phi \in C((-\infty, 0],[0,+\infty)): \phi(0)>0 \text { and } \phi \text { is bounded }\}, \quad i=1,2 .
$$

It is well known that system (1.9) has a unique solution $\left(x_{1}(t), x_{2}(t), u_{1}(t), u_{2}(t)\right)$ satisfying the initial condition (1.10) is said to be positive [34], and $x_{i}(t)>0, u_{i}(t)>0$ for all $i=1,2$.

Although feedback control can help avoid extinction, but the Allee effect of two species coexist according to it ecological significance. The aim of this paper is to explore the Allee effect to affect the permanence of the two-species competitive system with feedback controls and infinite delay by using the differential inequality theory and differential equation comparison principle.

\section{Permanence}

To investigate the persistence for system (1.9), we introduce the following lemmas. The following lemma is Lemma 3 of Oca and Vivas [10]. 
Lemma 2.1 Let $x: R \rightarrow R$ be a bounded nonnegative continuous function, and let $k$ : $[0,+\infty) \rightarrow(0,+\infty)$ be a continuous kernel such that $\int_{0}^{\infty} k(s) d s=1$. Then

$$
\begin{aligned}
\lim _{t \rightarrow+\infty} \inf x(t) & \leq \lim \inf _{t \rightarrow+\infty} \int_{-\infty}^{t} k(t-s) x(s) d s \\
& \leq \lim \sup _{t \rightarrow+\infty} \int_{-\infty}^{t} k(t-s) x(s) d s \\
& \leq \lim \sup _{t \rightarrow+\infty} x(t) .
\end{aligned}
$$

As a direct corollary of Lemma 1.4 of Chen [17], we have the following:

Lemma 2.2 If $a>0, b>0, \dot{x} \geq b-a x$, and $x(0)>0$, then we have

$$
\lim \inf _{t \rightarrow+\infty} x(t) \geq \frac{b}{a}
$$

If $a>0, b>0, \dot{x} \leq b-a x$, and $x(0)>0$, then we have

$$
\lim \sup _{t \rightarrow+\infty} x(t) \leq \frac{b}{a}
$$

As a direct corollary of Lemma 2.2 of Chen [35], we have the following:

Lemma 2.3 If $a>0, b>0, \dot{x} \geq x(b-a x)$, and $x(0)>0$, then we have

$$
\lim \inf _{t \rightarrow+\infty} x(t) \geq \frac{b}{a}
$$

If $a>0, b>0, \dot{x} \leq x(b-a x)$, and $x(0)>0$, then we have

$$
\lim \sup _{t \rightarrow+\infty} x(t) \leq \frac{b}{a}
$$

Now, we show that system (1.9) is permanent. From the view point of biology, this implies that the two species will always coexist at any time and any location of the inhabit domain, no matter what their Allee effect coefficients are.

Theorem 2.1 System (1.9) is permanent if there are positive constants $\delta, \theta(\theta>\delta>0)$ such that

$$
\begin{aligned}
& 0<\delta \leq \lim \inf _{t \rightarrow+\infty} x_{i}(t) \leq \lim \sup _{t \rightarrow+\infty} x_{i}(t) \leq \theta<+\infty, \\
& 0<\delta \leq \lim \inf _{t \rightarrow+\infty} u_{i}(t) \leq \lim \sup _{t \rightarrow+\infty} u_{i}(t) \leq \theta<+\infty
\end{aligned}
$$

for any solution $\left(x_{1}(t), x_{2}(t), u_{1}(t), u_{2}(t)\right)$ of $(1.9)$ with initial conditions $(1.8)$ and inequalities (1.2), (1.5) are satisfied. Here the kernels $k_{i}(s), i=1,2,3,4$, and $h_{i}(s), i=1,2$, are positive functions and satisfy (1.7). 
Proof Let $\left(x_{1}(t), x_{2}(t), u_{1}(t), u_{2}(t)\right)$ be any positive solution of system (1.9) with initial conditions (1.10). The ecological background and definition of the Allee effect shows that $1-\frac{A+c}{x_{1}(t)+c}<1$. Clearly, from the first and last equations of system (1.9) we get

$$
\begin{aligned}
x_{1}^{\prime}(t)= & x_{1}(t)\left[\left(a_{1}(t)-b_{11}(t) x_{1}(t)\right)\left(1-\frac{A+c}{x_{1}(t)+c}\right)\right. \\
& \left.-b_{12}(t) \int_{-\infty}^{t} k_{1}(t-s) x_{2}(s) d s-c_{1}(t) \int_{-\infty}^{t} h_{1}(t-s) u_{1}(s) d s\right] \\
\leq & x_{1}(t)\left[\left(a_{1}(t)-b_{11}(t) x_{1}(t)\right)\left(1-\frac{A+c}{x_{1}(t)+c}\right)\right] \\
\leq & x_{1}(t)\left(a_{1}(t)-b_{11}(t) x_{1}(t)\right) \\
\leq & x_{1}(t)\left(a_{1 M}-b_{11 L} x_{1}(t)\right), \\
u_{2}^{\prime}(t) \leq & f_{M}-e_{2 L} u_{2}(t) .
\end{aligned}
$$

Thus, from Lemmas 2.2 and 2.3 we have

$$
\begin{aligned}
& \lim \sup _{t \rightarrow+\infty} x_{1}(t) \leq \frac{a_{1 M}}{b_{11 L}} \stackrel{\text { def }}{=} \bar{x}_{1}, \\
& \lim \sup _{t \rightarrow+\infty} u_{2}(t) \leq \frac{f_{M}}{e_{2 L}} \stackrel{\text { def }}{=} \bar{u}_{2} .
\end{aligned}
$$

Evidently, from (2.1) and Lemma 2.1 we have

$$
\lim \sup _{t \rightarrow+\infty} \int_{-\infty}^{t} k_{2}(t-s) x_{1}(s) d s \leq \lim \sup _{t \rightarrow+\infty} x_{1}(t) \leq \bar{x}_{1}
$$

and

$$
\begin{gathered}
\lim \sup _{t \rightarrow+\infty} \int_{-\infty}^{t} h_{2}(t-s) u_{2}(s) d s \leq \lim \sup _{t \rightarrow+\infty} u_{2}(t) \leq \bar{u}_{2}, \\
\lim \sup _{t \rightarrow+\infty} \int_{-\infty}^{t} k_{3}(t-s) x_{1}(s) d s \leq \lim \sup _{t \rightarrow+\infty} x_{1}(t) \leq \bar{x}_{1} .
\end{gathered}
$$

For any small positive constant $\varepsilon>0$, from (2.4) it follows that there exists $T_{1}>0$ such that, for $t>T_{1}$,

$$
\begin{aligned}
& \int_{-\infty}^{t} h_{2}(t-s) u_{2}(s) d s \leq \bar{u}_{2}+\varepsilon \\
& \int_{-\infty}^{t} k_{3}(t-s) x_{1}(s) d s \leq \bar{x}_{1}+\varepsilon .
\end{aligned}
$$

Inequality (2.5), together with the second and third equations of system (1.9), leads to

$$
\begin{aligned}
& x_{2}^{\prime}(t) \leq x_{2}(t)\left[a_{2 M}+c_{2 M}\left(\bar{u}_{2}+\varepsilon\right)-b_{22 L} x_{2}(t)\right] \\
& u_{1}^{\prime}(t) \leq-e_{1 L} u_{1}(t)+d_{1 M}\left(\bar{x}_{1}+\varepsilon\right) .
\end{aligned}
$$


Again, applying Lemmas 2.2 and 2.3 to (2.6), we obtain

$$
\begin{aligned}
& \lim \sup _{t \rightarrow+\infty} x_{2}(t) \leq \frac{a_{2 M}+c_{2 M}\left(\bar{u}_{2}+\varepsilon\right)}{b_{22 L}}, \\
& \lim \sup _{t \rightarrow+\infty} u_{1}(t) \leq \frac{d_{1 M}\left(\bar{x}_{1}+\varepsilon\right)}{e_{1 L}} .
\end{aligned}
$$

Setting $\varepsilon \rightarrow 0$ in these inequalities, we get

$$
\begin{aligned}
& \lim \sup _{t \rightarrow+\infty} x_{2}(t) \leq \frac{a_{2 M}+c_{2 M} \bar{u}_{2}}{b_{22 L}} \stackrel{\text { def }}{=} \bar{x}_{2}, \\
& \lim \sup _{t \rightarrow+\infty} u_{1}(t) \leq \frac{d_{1 M} \bar{x}_{1}}{e_{1 L}} \stackrel{\text { def }}{=} \bar{u}_{1} .
\end{aligned}
$$

Thus, from (2.7) and Lemma 2.1 we easily have that

$$
\begin{aligned}
& \lim \sup _{t \rightarrow+\infty} \int_{-\infty}^{t} k_{i}(t-s) x_{2}(s) d s \leq \lim \sup _{t \rightarrow+\infty} x_{2}(t) \leq \bar{x}_{2}, \quad i=1,4 \\
& \lim \sup _{t \rightarrow+\infty} \int_{-\infty}^{t} h_{1}(t-s) u_{1}(s) d s \leq \lim \sup _{t \rightarrow+\infty} u_{1}(t) \leq \bar{u}_{1} .
\end{aligned}
$$

For any small positive constant $\varepsilon>0$, from (2.8) it follows that there exists $T_{2}>T_{1}$ such that, for $t>T_{2}$,

$$
\begin{aligned}
& \int_{-\infty}^{t} k_{i}(t-s) x_{2}(s) d s \leq \bar{x}_{2}+\varepsilon, \quad i=1,4, \\
& \int_{-\infty}^{t} h_{1}(t-s) u_{1}(s) d s \leq \bar{u}_{1}+\varepsilon .
\end{aligned}
$$

Hence, for $t>T_{2}$, inequality (2.9), together with the first and last equations of system (1.9), leads to

$$
\begin{aligned}
x_{1}^{\prime}(t)= & x_{1}(t)\left[\left(a_{1}(t)-b_{11}(t) x_{1}(t)\right)\left(1-\frac{A+c}{x_{1}(t)+c}\right)\right. \\
& \left.-b_{12}(t) \int_{-\infty}^{t} k_{1}(t-s) x_{2}(s) d s-c_{1}(t) \int_{-\infty}^{t} h_{1}(t-s) u_{1}(s) d s\right] \\
\geq & x_{1}(t)\left[\left(a_{1}(t)-b_{11}(t) x_{1}(t)\right)-\left(a_{1}(t)-b_{11}(t) x_{1}(t)\right)\left(\frac{A+c}{x_{1}(t)+c}\right)\right. \\
& \left.-b_{12 M}\left(\bar{x}_{2}+\varepsilon\right)-c_{1 M}\left(\bar{u}_{1}+\varepsilon\right)\right] \\
= & x_{1}(t)\left[\left(a_{1}(t)-b_{11}(t) x_{1}(t)\right)-a_{1}(t)\left(\frac{A+c}{x_{1}(t)+c}\right)+b_{11}(t) x_{1}(t)\left(\frac{A+c}{x_{1}(t)+c}\right)\right. \\
& \left.-b_{12 M}\left(\bar{x}_{2}+\varepsilon\right)-c_{1 M}\left(\bar{u}_{1}+\varepsilon\right)\right] \\
= & x_{1}(t)\left[\left(a_{1}(t)-b_{11}(t) x_{1}(t)\right)-a_{1}(t)\left(A-A+\frac{A+c}{x_{1}(t)+c}\right)+b_{11}(t) x_{1}(t)\right. \\
& \left.\cdot\left(\frac{A+c}{x_{1}(t)+c}\right)-b_{12 M}\left(\bar{x}_{2}+\varepsilon\right)-c_{1 M}\left(\bar{u}_{1}+\varepsilon\right)\right]
\end{aligned}
$$




$$
\begin{aligned}
= & x_{1}(t)\left[\left(a_{1}(t)-b_{11}(t) x_{1}(t)\right)-a_{1}(t) A+a_{1}(t)\left(A-\frac{A+c}{x_{1}(t)+c}\right)+b_{11}(t) x_{1}(t)\right. \\
& \left.\cdot\left(\frac{A+c}{x_{1}(t)+c}\right)-b_{12 M}\left(\bar{x}_{2}+\varepsilon\right)-c_{1 M}\left(\bar{u}_{1}+\varepsilon\right)\right] \\
= & x_{1}(t)\left[a_{1}(t)(1-A)-b_{11}(t) x_{1}(t)+a_{1}(t)\left(\frac{A x_{1}(t)+A c-A-c}{x_{1}(t)+c}\right)\right. \\
& \left.+b_{11}(t) x_{1}(t)\left(\frac{A+c}{x_{1}(t)+c}\right)-b_{12 M}\left(\bar{x}_{2}+\varepsilon\right)-c_{1 M}\left(\bar{u}_{1}+\varepsilon\right)\right] \\
= & x_{1}(t)\left[a_{1}(t)(1-A)+a_{1}(t)\left(\frac{A c-A-c}{x_{1}(t)+c}\right)-b_{12 M}\left(\bar{x}_{2}+\varepsilon\right)-c_{1 M}\left(\bar{u}_{1}+\varepsilon\right)\right. \\
& \left.-b_{11}(t) x_{1}(t)+\left(\frac{a_{1}(t) A}{x_{1}(t)+c}\right) x_{1}(t)+b_{11}(t) x_{1}(t)\left(\frac{A+c}{x_{1}(t)+c}\right)\right] \\
\geq & x_{1}(t)\left[a_{1 L}(1-A)+a_{1 L}\left(\frac{A c-A-c}{\bar{x}_{1}+c}\right)-b_{12 M}\left(\bar{x}_{2}+\varepsilon\right)-c_{1 M}\left(\bar{u}_{1}+\varepsilon\right)\right. \\
& \left.-\left(b_{11 M}-\left(\frac{a_{1 L} A}{\bar{x}_{1}+c}\right)-b_{11 L}\left(\frac{A+c}{\bar{x}_{1}+c}\right)\right) x_{1}(t)\right] .
\end{aligned}
$$

Here, from (1.5), without loss of generality, we may choose $\varepsilon$ small enough such that

$$
\frac{a_{1 L}(1-A)+a_{1 L} b_{11 L}\left(\frac{A c-A-c}{a_{1 M}+c b_{11 L}}\right)-b_{12 M}\left(\frac{e_{2 L} a_{2 M}+c_{2 M} f_{M}}{e_{2 L} b_{22 L}}+\varepsilon\right)-c_{1 M}\left(\frac{d_{1 M} a_{1 M}}{e_{1 L} b_{11 L}}+\varepsilon\right)}{b_{11 M}-\left(\frac{b_{11 L} a_{1 L} A}{a_{1 M}+c b_{11 L}}\right)-b_{11 L}^{2}\left(\frac{A+c}{a_{1 M}+c b_{11 L}}\right)}>0 .
$$

Integrating this inequality, we get

$$
\frac{a_{1 L}(1-A)+a_{1 L}\left(\frac{A c-A-c}{\bar{x}_{1}+c}\right)-b_{12 M}\left(\bar{x}_{2}+\varepsilon\right)-c_{1 M}\left(\bar{u}_{1}+\varepsilon\right)}{b_{11 M}-\left(\frac{a_{1 L} A}{\bar{x}_{1}+c}\right)-b_{11 L}\left(\frac{A+c}{\bar{x}_{1}+c}\right)}>0 .
$$

Also, because of

$$
u_{2}^{\prime}(t) \geq f_{L}-d_{2 M}\left(\bar{x}_{2}+\varepsilon\right)-e_{2 M} u_{2}(t)
$$

as a conclusion, we have

$$
f_{L}-\frac{d_{2 M} a_{2 M} e_{2 L}+d_{2 M} c_{2 M} f_{M}}{b_{22 L} e_{2 L}}-d_{2 M} \varepsilon>0
$$

Integrating the above inequality, we get

$$
f_{L}-d_{2 M}\left(\bar{x}_{2}+\varepsilon\right)>0 .
$$

Thus, applying Lemmas 2.2 and 2.3, we have

$$
\begin{aligned}
& \lim \inf _{t \rightarrow+\infty} x_{1}(t) \geq \frac{a_{1 L}(1-A)+a_{1 L}\left(\frac{A c-A-c}{\bar{x}_{1}+c}\right)-b_{12 M}\left(\bar{x}_{2}+\varepsilon\right)-c_{1 M}\left(\bar{u}_{1}+\varepsilon\right)}{b_{11 M}-\left(\frac{a_{1 L} A}{\bar{x}_{1}+c}\right)-b_{11 L}\left(\frac{A+c}{\bar{x}_{1}+c}\right)}>0, \\
& \lim \inf _{t \rightarrow+\infty} u_{2}(t) \geq \frac{f_{L}-d_{2 M}\left(\bar{x}_{2}+\varepsilon\right)}{e_{2 M}}>0 .
\end{aligned}
$$


Letting $\varepsilon \rightarrow 0$ in this inequality leads to

$$
\begin{aligned}
& \lim \inf _{t \rightarrow+\infty} x_{1}(t) \geq \frac{a_{1 L}(1-A)+a_{1 L}\left(\frac{A c-A-c}{\bar{x}_{1}+c}\right)-b_{12 M} \bar{x}_{2}-c_{1 M} \bar{u}_{1}}{b_{11 M}-\left(\frac{a_{1 L} A}{\bar{x}_{1}+c}\right)-b_{11 L}\left(\frac{A+c}{\bar{x}_{1}+c}\right)} \stackrel{\text { def }}{=} \underline{x}_{1}>0, \\
& \lim \inf _{t \rightarrow+\infty} u_{2}(t) \geq \frac{f_{L}-d_{2 M} \bar{x}_{2}}{e_{2 M}} \stackrel{\text { def }}{=} \underline{u}_{2}>0 .
\end{aligned}
$$

From (2.11) and Lemma 2.1 we obtain

$$
\begin{aligned}
& \lim \inf _{t \rightarrow+\infty} \int_{-\infty}^{t} h_{2}(t-s) u_{2}(s) d s \geq \lim \inf _{t \rightarrow+\infty} u_{2}(t) \geq \underline{u}_{2}, \\
& \lim \inf _{t \rightarrow+\infty} \int_{-\infty}^{t} k_{3}(t-s) x_{1}(s) d s \geq \lim \inf _{t \rightarrow+\infty} x_{1}(t) \geq \underline{x}_{1} .
\end{aligned}
$$

For any small positive constant $\varepsilon>0$, from the second inequality of (1.5) we may choose $\varepsilon$ small enough such that

$$
\varepsilon<\frac{1}{2} \min \left\{\underline{u}_{2}, \underline{x}_{1}\right\}
$$

and

$$
a_{2 L}-b_{21 M}\left(\bar{x}_{1}+\varepsilon\right)+c_{2 L}\left(\underline{u}_{2}+\varepsilon\right)>0 \text {. }
$$

For such $\varepsilon>0$, from (2.3) and (2.12) it follows that there exists $T_{3}>T_{2}>0$ such that, for $t>T_{3}$,

$$
\begin{aligned}
& \int_{-\infty}^{t} k_{2}(t-s) x_{1}(s) d s \leq \bar{x}_{1}+\varepsilon \\
& \int_{-\infty}^{t} h_{2}(t-s) u_{2}(s) d s \geq \underline{u}_{2}-\varepsilon \\
& \int_{-\infty}^{t} k_{3}(t-s) x_{1}(s) d s \geq \underline{x}_{1}-\varepsilon
\end{aligned}
$$

Inequality (2.13), together with the second and third equations of system (1.9), leads to

$$
\begin{aligned}
& x_{2}^{\prime}(t) \geq x_{2}(t)\left[a_{2 L}-b_{21 M}\left(\bar{x}_{1}+\varepsilon\right)+c_{2 L}\left(\underline{u}_{2}-\varepsilon\right)-b_{22 M} x_{2}(t)\right], \\
& u_{1}^{\prime}(t) \geq-e_{1 M} u_{1}(t)+d_{1 L}\left(\underline{x}_{1}-\varepsilon\right) .
\end{aligned}
$$

Again, applying Lemmas 2.2 and 2.3 to (2.6), we obtain

$$
\begin{aligned}
& \lim \inf _{t \rightarrow+\infty} x_{2}(t) \geq \frac{a_{2 L}-b_{21 M}\left(\bar{x}_{1}+\varepsilon\right)+c_{2 L}\left(\underline{u}_{2}-\varepsilon\right)}{b_{22 M}}>0, \\
& \lim \inf _{t \rightarrow+\infty} u_{1}(t) \geq \frac{d_{1 L}\left(\underline{x}_{1}-\varepsilon\right)}{e_{1 M}}>0 .
\end{aligned}
$$


There exists small $\varepsilon \rightarrow 0$ such that

$$
\begin{aligned}
& \lim \inf _{t \rightarrow+\infty} x_{2}(t) \geq \frac{a_{2 L}-b_{21 M} \bar{x}_{1}+c_{2 L} \underline{u}_{2}}{b_{22 M}} \stackrel{\text { def }}{=} \underline{x}_{2}>0, \\
& \lim \inf _{t \rightarrow+\infty} u_{1}(t) \geq \frac{d_{1 L} \underline{x}_{1}}{e_{1 M}} \stackrel{\text { def }}{=} \underline{u}_{1}>0 .
\end{aligned}
$$

Now, let $\delta=\frac{1}{2} \min \left\{\underline{x}_{i}, \underline{u}_{i}, i=1,2\right\}$ and $\theta=2 \max \left\{\bar{x}_{i}, \bar{u}_{i}, i=1,2\right\}$. Then $\delta, \theta$ are independent of any positive solution of system (1.9). Also, from (2.2), (2.7), (2.11), and (2.15) it immediately follows that

$$
\begin{aligned}
& 0<\delta \leq \lim \inf _{t \rightarrow+\infty} x_{i}(t) \leq \lim \sup _{t \rightarrow+\infty} x_{i}(t) \leq \theta<+\infty, \\
& 0<\delta \leq \lim \inf _{t \rightarrow+\infty} u_{i}(t) \leq \lim \sup _{t \rightarrow+\infty} u_{i}(t) \leq \theta<+\infty .
\end{aligned}
$$

The above inequality shows that system (1.9) is permanent.

\section{Periodic solution}

Theorem 3.1 ([14]) Assume (2.2), (2.7), (2.11) hold. Then

$$
K=\left\{\left(x_{1}(t), x_{2}(t), u_{1}(t), u_{2}(t)\right): x_{i L} \leq x_{i}(t) \leq x_{i M}, u_{i L} \leq u_{i}(t) \leq u_{i M}, i=1,2\right\}
$$

is an ultimately bounded region of system (1.9).

In this section, we suppose that system (1.9) is an $\omega$-period system. For $X=\left(x_{1}, x_{2}, u_{1}\right.$, $\left.u_{2}\right) \in R^{4}$, we define the norm as

$$
\|X\|=\left|x_{1}\right|+\left|x_{2}\right|+\left|u_{1}\right|+\left|u_{2}\right|
$$

We can define a Poincaré map on $K \subset R^{4}$ (the Banach space with previously defined norm), and by the Brower fixed-point theorem and Theorem 3.1 we obtain the following:

Theorem 3.2 ([14]) Assume that (2.2), (2.7), (2.11) hold. Then the $\omega$-period system (1.9) has at least one $\omega$-period solution.

Theorem 3.3 Assume that (2.2), (2.7), (2.11) hold and that there exists positive constants $p_{i}, q_{i}, l_{i}, m_{i}(i=1,2)$ such that

$$
\begin{aligned}
& q_{1} d_{1 L} \underline{x}_{1}>p_{1}\left(-b_{11 L}-\frac{b_{11 L}(A+c)}{\underline{x}+c}\right)+p_{2} b_{21 M} \bar{x}_{1}, \\
& p_{2} b_{22 L}>p_{1} b_{12 M \underline{x}_{2}}+q_{2} d_{2 M} \underline{x}_{2}, \quad q_{1} e_{1 L}>p_{1} c_{1 L} \underline{u}_{1}, \quad q_{2} e_{2 L}>p_{2} c_{2 M} \bar{u}_{2} .
\end{aligned}
$$

Then system (1.9) has a periodic solution that is globally attractive.

Proof Suppose $W(t)=\left\{x_{1}(t), x_{2}(t), u_{1}(t), u_{2}(t)\right\}$ is any solution of system (1.9) with positive initial value and $W_{0}(t)=\left\{x_{10}(t), x_{20}(t), u_{10}(t), u_{20}(t)\right\}$ is a strictly positive periodic solution of system (1.9). 
Consider the Lyapunov function $V(t)$ as follows:

$$
V(t)=\sum_{j=1}^{2}\left[p_{j}\left|\ln x_{j}(t)-\ln x_{j 0}(t)\right|+q_{j}\left|u_{j}(t)-u_{j 0}(t)\right|\right]
$$

Calculating the right derivative $D^{+} V$ of $V$ along the solution of (1.9), we get

$$
\begin{aligned}
& D^{+} V(t)=D^{+}\left\{\sum_{j=1}^{2}\left[p_{j}\left|\ln x_{j}(t)-\ln x_{j 0}(t)\right|+q_{j}\left|u_{j}(t)-u_{j 0}(t)\right|\right]\right\} \\
& =\left\{p_{1}\left(-b_{11}(t)+\frac{\left(a_{1}(t)-b_{11}(t)(A+c)\right)}{x_{1}(t)+c}\right)-p_{2} b_{21}(t) D^{+}\left(\int_{-\infty}^{t} k_{2}(t-s) x_{1}(s) d s\right)\right. \\
& \left.+q_{1} d_{1}(t) D^{+}\left(\int_{-\infty}^{t} k_{3}(t-s) x_{1}(s) d s\right)\right\} \cdot\left|x_{1}-x_{10}\right| \\
& +\left\{-p_{1} b_{12}(t) D^{+}\left(\int_{-\infty}^{t} k_{1}(t-s) x_{2}(s) d s\right)\right. \\
& \left.-p_{2} b_{22}(t)-q_{2} d_{2}(t) D^{+}\left(\int_{-\infty}^{t} k_{4}(t-s) x_{2}(s) d s\right)\right\} \cdot\left|x_{2}-x_{20}\right| \\
& +\left\{-p_{1} c_{1}(t) D^{+}\left(\int_{-\infty}^{t} h_{1}(t-s) u_{1}(s) d s\right)-q_{1} e_{1}(t)\right\} \cdot\left|u_{1}-u_{10}\right| \\
& +\left\{p_{2} c_{2}(t) D^{+}\left(\int_{-\infty}^{t} h_{2}(t-s) u_{2}(s) d s\right)-q_{2} e_{2}(t)\right\} \cdot\left|u_{2}-u_{20}\right| \\
& \leq\left\{p_{1}\left(-b_{11 L}-\frac{b_{11 L}(A+c)}{\underline{x}+c}\right)-p_{2} b_{21 L} D^{+}\left(\int_{-\infty}^{t} k_{2}(t-s) x_{1}(s) d s\right)+q_{1} d_{1 M}\right. \\
& \left.\cdot D^{+}\left(\int_{-\infty}^{t} k_{3}(t-s) x_{1}(s) d s\right)\right\} \cdot\left|x_{1}-x_{10}\right| \\
& +\left\{-p_{1} b_{12 L} D^{+}\left(\int_{-\infty}^{t} k_{1}(t-s) x_{2}(s) d s\right)-p_{2} b_{22 L}\right. \\
& \left.-q_{2} d_{2 L} D^{+}\left(\int_{-\infty}^{t} k_{4}(t-s) x_{2}(s) d s\right)\right\} \cdot\left|x_{2}-x_{20}\right| \\
& +\left\{-p_{1} c_{1 L} D^{+}\left(\int_{-\infty}^{t} h_{1}(t-s) u_{1}(s) d s\right)-q_{1} e_{1 L}\right\} \\
& \cdot\left|u_{1}-u_{10}\right|+\left\{p_{2} c_{2 M} D^{+}\left(\int_{-\infty}^{t} h_{2}(t-s) u_{2}(s) d s\right)-q_{2} e_{2 L}\right\} \cdot\left|u_{2}-u_{20}\right| \\
& \leq\left\{p_{1}\left(-b_{11 L}-\frac{b_{11 L}(A+c)}{\underline{x}+c}\right)+p_{2} b_{21 M}\left(\bar{x}_{1}+\varepsilon\right)-q_{1} d_{1 L}\left(\underline{x}_{1}-\varepsilon\right)\right\} \cdot\left|x_{1}-x_{10}\right| \\
& +\left\{p_{1} b_{12 M}\left(\underline{x}_{2}-\varepsilon\right)-p_{2} b_{22 L}+q_{2} d_{2 M}\left(\underline{x}_{2}-\varepsilon\right)\right\} \cdot\left|x_{2}-x_{20}\right| \\
& +\left\{p_{1} c_{1 L}\left(\underline{u}_{1}-\varepsilon\right)-q_{1} e_{1 L}\right\} \\
& \cdot\left|u_{1}-u_{10}\right|+\left\{p_{2} c_{2 M}\left(\bar{u}_{2}+\varepsilon\right)-q_{2} e_{2 L}\right\} \cdot\left|u_{2}-u_{20}\right| \\
& =\left\{p_{1}\left(-b_{11 L}-\frac{b_{11 L}(A+c)}{\underline{x}+c}\right)+p_{2} b_{21 M} \bar{x}_{1}-q_{1} d_{1 L} \underline{x}_{1}\right\} \cdot\left|x_{1}-x_{10}\right| \\
& +\left\{p_{1} b_{12 M} \underline{x}_{2}-p_{2} b_{22 L}+q_{2} d_{2 M} \underline{x}_{2}\right\} \cdot\left|x_{2}-x_{20}\right|+\left\{p_{1} c_{1 L} \underline{u}_{1}-q_{1} e_{1 L}\right\} \\
& \cdot\left|u_{1}-u_{10}\right|+\left\{p_{2} c_{2 M} \bar{u}_{2}-q_{2} e_{2 L}\right\} \cdot\left|u_{2}-u_{20}\right|(\varepsilon=0)
\end{aligned}
$$




$$
\begin{aligned}
& =-m_{1}\left|x_{1}-x_{10}\right|-m_{2}\left|x_{2}-x_{20}\right|-l_{1}\left|u_{1}-u_{10}\right|-l_{2}\left|u_{2}-u_{20}\right| \\
& \leq-m \sum_{i=1}^{2}\left[\left|x_{i}-x_{i 0}\right|+\left|u_{i}-u_{i 0}\right|\right] \\
& \leq-m\left\|W(t)-W_{0}(t)\right\|,
\end{aligned}
$$

where

$$
\begin{aligned}
& m=\min \left\{m_{i}, l_{i}, i=1,2\right\}, \quad m_{1}=q_{1} d_{1 L} \underline{x}_{1}-p_{1}\left(-b_{11 L}-\frac{b_{11 L}(A+c)}{\underline{x}+c}\right)-p_{2} b_{21 M} \bar{x}_{1}, \\
& m_{2}=\left\{p_{2} b_{22 L}-p_{1} b_{12 M} \underline{x}_{2}-q_{2} d_{2 M} \underline{x}_{2}\right\}, \quad l_{1}=\left\{q_{1} e_{1 L}-p_{1} c_{1 L} \underline{u}_{1}\right\}, \\
& l_{2}=\left\{q_{2} e_{2 L}-p_{2} c_{2 M} \bar{u}_{2}\right\} .
\end{aligned}
$$

Then $V(t)$ is decreasing on $[0, \infty)$; thus $0 \leq V(t) \leq V(0)$, and

$$
\lim _{t \rightarrow \infty} V(t)=V^{*} \geq 0, \quad\left|\ln x_{i}\right| \leq\left|\ln x_{i}-\ln x_{i 0}\right|+\left|\ln x_{i 0}\right| \leq V(t)+\left|\ln x_{i 0}\right|
$$

So $x_{i}(i=1,2)$ is bounded. By the same reason, we have that $u_{i}(i=1,2)$ is bounded. Hence, $W(t)$ is bounded; by the usual mean value theorem there are positive constants $\beta_{0}$, $\beta_{1}$ such that

$$
\frac{1}{\beta_{0}}\left|x_{i}-x_{i 0}\right|+\left|u_{i}-u_{i 0}\right| \leq\left|\ln x_{i}-\ln x_{i 0}\right|+\left|u_{i}-u_{i 0}\right| \leq \frac{1}{\beta_{1}}\left|x_{i}-x_{i 0}\right|+\left|u_{i}-u_{i 0}\right| \quad(i=1,2) .
$$

From this it follows that there are positive constants $\beta_{2}, \beta_{3}$ such that

$$
\frac{1}{\beta_{3}}\left\|W(t)-W_{0}(t)\right\| \leq V(t) \leq \frac{1}{\beta_{2}}\left\|W(t)-W_{0}(t)\right\|
$$

And thus $D^{+} V(t) \leq-m \beta_{2} V(t)$. We claim that $V^{*}=0$. Otherwise, $V^{*}>0$, and we have $V(t) \geq V^{*}>0$, so $D^{+} V(t) \leq-m \beta_{2} V^{*}$, and then

$$
V(t) \leq V(0)-m \beta_{2} V^{*} \rightarrow-\infty \quad(t \rightarrow \infty)
$$

This contradicts with positivity of $V(t)$, so $V^{*}=0$, and we have

$$
0 \leq \lim _{t \rightarrow \infty}\left\|W(t)-W_{0}(t)\right\| \leq \lim _{t \rightarrow \infty} \beta_{3} V(t)=0
$$

And thus the periodic solution $W_{0}(t)$ is globally attractive.

\section{Discussion}

In [14], it was shown that, for infinite delay ecosystem, feedback controls can avoid the extinction of the species without the Allee effect, and the upper bound of $x_{1}(t)$ is $\frac{a_{1 M}}{b_{11 L}}$, whereas the lower bound is $\frac{a_{1 L}-b_{12 M} \bar{x}_{2}-c_{1 M} \bar{u}_{1}}{b_{11 M}}$. This paper introduces the Allee effect, and we easily prove that the upper bound of $x_{1}(t)$ with Allee effect is invariant, and the lower 
bound is $\frac{a_{1 L}(1-A)+a_{1 L}\left(\frac{A c-A-c}{\bar{x}_{1}+c}\right)-b_{12 M} \bar{x}_{2}-c_{1 M} \bar{u}_{1}}{b_{11 M}-\left(\frac{a_{1 L} A}{\bar{x}_{1}+c}\right)-b_{11 L}\left(\frac{A+c}{x_{1}+c}\right)}$. If $A=c=0$, then we prove that the lower bound of $x_{1}(t)$ with Allee effect is equal to the lower bound without Allee effect. By contrast, the lower bound of $x_{1}(t)$ becomes greater, and then our analysis reveals that the Allee effect increases the risk of one population extinction and is unfavorable for two species to achieve persistence.

As well known, the periodic solution is an important topic in the study of dynamics for differential equations and population models. Weng [36] and Liu [37] studied the periodic solution to the two-species competitive system with feedback controls. Peng [38], Seifert [39], and Muhammadhaji [40] studied the periodic solution to the two-species competitive system with infinite delay and pure delays. Moreover, in this paper, we obtain the periodic solution of system (1.9), which overcomes some limitations and deficiency of the existing ones.

\section{Funding}

This work was supported by the Fundamental Research Funds for the Central Universities (31920180116, 31920180044, 31920170072), the National Natural Science Foundation of China (31260098, 31560127), the Program for Yong Talent of State Ethnic Affairs Commission of China (No. [2014]121), and Gansu Provincial first-class discipline program of Northwest Minzu University.

\section{Competing interests}

The authors declare that they have no competing interests.

\section{Authors' contributions}

All authors contributed equally and significantly in this paper. All authors read and approved the final manuscript.

\section{Author details}

${ }^{1}$ School of Mathematics and Computer Science, Northwest Minzu University, Lanzhou, People's Republic of China.

²Experimental Center, Northwest Minzu University, Lanzhou, People's Republic of China.

\section{Publisher's Note}

Springer Nature remains neutral with regard to jurisdictional claims in published maps and institutional affiliations.

Received: 12 June 2018 Accepted: 21 October 2018 Published online: 26 October 2018

\section{References}

1. Ahmad, S.: On the nonautonomous Volterra-Lotka competition equations. Proc. Am. Math. Soc. 117(1), 119-204 (1993)

2. Teng, Z.D.: On the nonautonomous Lotka-Volterra N-species competing systems. Appl. Math. Comput. 114(2-3), 175-185 (2000)

3. Teng, Z.D.: On the permanence and extinction in nonautonomous Lotka-Volterra competitive systems with delays. Acta Math. Sin. 44(2), 293-306 (2001)

4. Teng, Z.D., Yu, Y.H.: Some new results of nonautonomous Lotka-Volterra competitive systems with delays. J. Math. Anal. Appl. 241, 254-275 (2000)

5. Zhao, J.D., Jiang, J.F.: Average conditions for permanence and extinction in nonautonomous Lotka-Volterra system. J. Math. Anal. Appl. 299, 663-675 (2004)

6. Chen, F.D.: Average conditions for permanence and extinction in nonautonomous Gilpin-Ayala competition model. Nonlinear Anal., Real World Appl. 7(4), 895-915 (2006)

7. Chen, F.D.: Some new results on the permanence and extinction of nonautonomous Gilpin-Ayala type competition model with delays. Nonlinear Anal., Real World Appl. 7(5), 1205-1222 (2006)

8. Lisena, B.: Competitive exclusion in a periodic Lotka-Volterra system. Appl. Math. Comput. 177(2), 761-768 (2006)

9. Muroya, Y.: Boundedness and partial survival of species in nonautonomous Lotka-Volterra systems. Nonlinear Anal., Real World Appl. 6(2), 263-272 (2005)

10. Oca, F.M.D., Vivas, M.: Extinction in a two dimensional Lotka-Volterra system with infinite delay. Nonlinear Anal., Real World Appl. 7(5), 1042-1047 (2006)

11. Tineo, A.: Asymptotic behavior of positive solutions of the nonautonomous Lotka-Volterra competition equations. Differ. Integral Equ. 6, 419-457 (1993)

12. Hirsch, W., Hanisch, H., Gabriel, J.: Differential equation models of some parasitic infection-methods for the study of asymptotic behavior. Commun. Pure Appl. Math. 38, 733-753 (1985)

13. Xiao, Y.N., Tang, S.Y., Chen, J.F.: Permanence and periodic solution in competitive system with feedback controls. Math. Comput. Model. 27(6), 33-37 (1998)

14. Chen, F.D., Li, Z., Huang, Y.: Note on the permanence of a competitive system with infinite delay and feedback controls. Nonlinear Anal., Real World Appl. 8(2), 680-687 (2007) 
15. Chen, F.D.: Global asymptotic stability in $n$-species nonautonomous Lotka-Volterra competitive systems with infinite delays and feedbackcontrol. Appl. Math. Comput. 170(2), 1452-1468 (2005)

16. Chen, F.D.: Positive periodic solutions of neutral Lotka-Volterra system with feedback control. Appl. Math. Comput. 162(3), 1279-1302 (2005)

17. Chen, F.D.: The permanence and global attractivity of Lotka-Volterra competition system with feedback controls. Nonlinear Anal., Real World Appl. 7(1), 133-143 (2006)

18. Yang, Z., Cao, J.: Sufficient conditions for the existence of positive periodic solutions of a class of neutral delay models. Appl. Math. Comput. 142(1), 123-142 (2003)

19. Chen, F.D., Chen, X.X., Cao, J.D., Chen, A.P.: Positive periodic solutions of a class of non-autonomous single species population model with delays and feedback control. Acta Math. Sin. 21(6), 1319-1336 (2005)

20. Fan, M., Wang, K., Wong, P.R., Agarwal, R.: Periodicity and stability in periodic $n$-species Lotka-Volterra competition system with feedback controlsand deviating arguments. Acta Math. Sin. 19(4), 801-822 (2003)

21. Gopalsamy, K., Weng, P.X.: Global attractivity in a competition system with feedback controls. Comput. Math. Appl. 45(4-5), 665-676 (2003)

22. Huang, Z.K., Chen, F.D.: Almost periodic solution of two species model with feedback regulation and infinite delay. Chin. J. Eng. Math. 21(1), 33-40 (2004)

23. Huo, H.F., Li, W.T.: Positive periodic solutions of a class of delay differential system with feedback control. Appl. Math. Comput. 148(1), 35-46 (2004)

24. Li, Y.K.: Positive periodic solutions for a periodic neutral differential equation with feedback control. Nonlinear Anal., Real World Appl. 6(1), 145-154 (2005)

25. Li, W.T., Wang, L.L.: Existence and global attractivity of positive periodic solutions of functional differential equations with feedback control. J. Comput. Appl. Math. 180(2), 293-309 (2005)

26. Weng, P.: Existence and global stability of positive periodic solution of periodic integro-differential systems with feedback controls. Comput. Math. Appl. 40(6-7), 747-759 (2000)

27. Yin, F.Q., Li, Y.K.: Positive periodic solutions of a single species model with feedback regulation and distributed time delay. Appl. Math. Comput. 153(2), 475-484 (2004)

28. Brânzei, R., Tijs, S.: A delayed predator-prey model with strong Allee effect in prey population growth. Nonlinear Dyn. 68(1-2), 23-42 (2012)

29. Tobin, P.C., Berec, L., Liebhold, A.M.: Exploiting Allee effects for managing biological invasions. Ecol. Lett. 14(6), 615-624 (2011)

30. Jian, Z.: Global qualitative analysis of a predator-prey system with Allee effect on the preyspecies. Math. Comput. Simul. 94, 33-54 (2013)

31. Sun, G.Q.: Mathematical modeling of population dynamics with Allee effect. Nonlinear Dyn. 85(1), 1-12 (2016)

32. Jang, R.J.: On the Lotka-Volterra competition system with Allee effects. Comput. Appl. Math. 32(1), 179-189 (2013)

33. Assas, L., Elaydi, S., Kwessi, E., Livadiotis, G., Ribble, D.: Competition models with Allee effects. J. Differ. Equ. Appl. 20(8), $1127-1145(2014)$

34. Kiguradze, I., P P̊ža, B.: On boundary value problems for systems of linear functional differential equations. Czechoslov Math. J. 47(2), 341-373 (1997)

35. Chen, F.D.: On a nonlinear non-autonomous predator-prey model with diffusion and distributed delay. J. Comput. Appl. Math. 180(1), 33-49 (2005)

36. Weng, P.: Existence and global stability of positive periodic solution of periodic integro differential systems with feedback controls. Comput. Math. Appl. 40(6), 747-759 (2000)

37. Liu, Z.J.: Persistence and periodic solution in two species competitive system with feedback controls. J. Biomath. 6(10), 2168-2176 (2002)

38. Seifert, G.: Almost periodic solutions for delay-differential equations with infinite delays. J. Differ. Equ. 41(3), 416-425 (1981)

39. Feng, C.: Almost periodic solutions for some integro differential equations with infinite delay. Appl. Math. Lett. 18(3), 245-252 (2005)

40. Muhammadhaji, A., Mahemuti, R., Teng, Z.: Periodic solutions for $n$-species Lotka-Volterra competitive systems with pure delays. Appl. Spectrosc. 69(7), 865 (2015)

\section{Submit your manuscript to a SpringerOpen ${ }^{\circ}$ journal and benefit from:}

- Convenient online submission

- Rigorous peer review

- Open access: articles freely available online

- High visibility within the field

- Retaining the copyright to your article

Submit your next manuscript at $\gg$ springeropen.com 